\title{
Estudo da microestrutura e da microdureza das cerâmicas piezoelétricas tipos PZT I e III utilizadas em transdutores eletroacústicos
}

\section{Study of the microestructure and the hardness of PZT piezoelectric ceramics types I and III used in electroacoustic transducers}

\author{
${ }^{1}$ Ricardo de Freitas Cabral \\ ${ }^{2}$ Lucas Mendes Itaboray \\ ${ }^{3}$ Anna Paula de Oliveira Santos
}

\footnotetext{
1 UniFOA - Professor responsável doutor, Centro Universitário Geraldo Di Biase - UGB.

2 Graduando Engenharia Elétrica, UniFOA.

3 Graduanda em Engenharia Ambiental, UniFOA
}

\section{RESUMO}

O domínio do processamento de cerâmica eletrônica do tipo piezoelétrica, a partir de matéria-prima importada, levou à produção de cerâmicas com $97 \%$ da densidade teórica, de microestruturas homogêneas, com grande potencial para aplicações em dispositivos piezoelétricos, como por exemplo, os transdutores eletroacústicos. Porém, a produção de cerâmica eletrônica do tipo piezoelétrica nacional ainda não é capaz de ter como matéria-prima o Titanato Zirconato de Chumbo (PZT) 100\% produzido no Brasil. Dessa forma, utiliza-se, para essa produção, o fornecimento de Óxido de Zircônio importado. Neste trabalho, ambos os pós de PZT tipos I e III, importados, foram prensados uniaxialmente a 70 MPa e sinterizados a 1200 e $1250^{\circ} \mathrm{C}$ por $3 \mathrm{~h}$. Foram realizadas medidas de dureza por micro-indentação, análises de Difração de raios X e Microscopia Eletrônica de Varredura. A dureza do PZT I foi de 393 HV.

\section{Palavras-chave}

PZT; Processamento; dureza; Transdutores Eletroacústicos

\section{ABSTRACT}

The field of electronic processing of the ceramic piezoelectric type imported powdered led to the production of ceramics with $97 \%$ of theoretical density, homogeneous microestructure with great potential for applications in piezoelectric devices such as electroacoustic transducers. However, the production of electronic ceramics National piezoelectric type is not yet able to have as raw material zirconate titanate Lead (PZT) $100 \%$ made in Brazil. Thus, this is used for supply of domestic production, the zirconium oxide. In this work, both post PZT types I and III, imported, were uniaxially pressed at $70 \mathrm{MPa}$ and sintered at 1200 and $1250^{\circ} \mathrm{C}$ for 3 hours. Hardness measurements were performed by microindentação, diffraction analysis and X-ray Scanning Electron Microscopy. The hardness of PZT I was $393 \mathrm{HV}$.

\section{Keywords}

PZT; Processing; Properties Piezo; Electroacoustic Transducers

\section{Como você deve citar?}

CABRAL, Ricardo de Freitas; ITABORAY, Lucas Mendes; SANTOS, Anna Paula de Oliveira. Estudo da microestrutura e da microdureza das cerâmicas piezoelétricas tipos PZT I e III utilizadas em transdutores eletroacústicos. Cadernos UniFOA, Volta Redonda, n. 27, p. 17-24, dez. 2015. 


\section{INTRODUÇÃO}

Após a primeira guerra mundial, devido à dificuldade de se excitar transdutores construídos com cristais de quartzo por demandarem geradores de alta tensão, iniciou-se o desenvolvimento de materiais piezoelétricos sintéticos. Esses esforços levaram à descoberta e aperfeiçoamento nas décadas de 40 e 50, das cerâmicas piezoelétricas de Titanato de Bário, pela então URSS e Japão, e das cerâmicas piezoelétricas de Titanato Zirconato de Chumbo (PZT's), pelos EUA (KOUR, 2013; YANG, 1998; KHALID, 2011).

O desenvolvimento das cerâmicas piezoelétricas foi revolucionário. Além de apresentarem meIhores propriedades que os cristais, após "polarizadas", também oferecem geometrias e dimensões flexíveis, por serem fabricadas por meio da sinterização de pós cerâmicos conformados via prensagem. Atualmente, as cerâmicas piezoelétricas tipo PZT, em suas diversas variações, são as cerâmicas predominantes no mercado. Também se pode encontrar outros materiais, como por exemplo, o PT (PbTiO3) e o $\mathrm{PMN}(\mathrm{Pb}(\mathrm{Mg} 1 / 3 \mathrm{Nb} 2 / 3) 03)$, utilizados em dispositivos que exigem propriedades especiais e muito específicas, como transdutores para alta temperatura (YANG, 1998; FESENKO, 2003).

As Cerâmicas Piezoelétricas apresentam grande flexibilidade de formato e de propriedades, sendo largamente utilizadas na fabricação de equipamentos ultrassônicos de potência, de ensaios não destrutivos e de atuadores. Os materiais piezoelétricos também são utilizados em detonadores de impacto, geradores de faíscas (magic clicks), nebulizadores, atuadores, posicionadores e transformadores, entre outras aplicações. (KHALID, 2011; FESENKO, 2003).

O objetivo deste trabalho foi o de desenvolver material piezoelétrico do tipo PZT, quimicamente estável e denso, a fim de ser empregado como transdutores eletroacústicos.

\section{PROCEDIMENTOS EXPERIMENTAIS}

\subsection{Processamento cerâmico}

Os pós de PZT utilizados foram o tipo I (SP-4) dopado com estrôncio, produzido pela empresa Sparkler Ceramics Pvt. Ltda. e o tipo III (EC-69) dopado com ferro, produzido pela EDO Corporation.

Os corpos verdes foram moldados por prensagem uniaxial a $70 \mathrm{MPa}$ em matriz de 1,998 cm de diâmetro em três estágios: 0,7; 1,4 e 2,1 Tons, com 10s de aplicação de carga para cada estágio, numa prensa de bancada da marca MARCON.

Os corpos de prova foram sinterizados a 1200 e $1250^{\circ} \mathrm{C}$, com taxa de aquecimento e resfriamento de $4{ }^{\circ} \mathrm{C} / \mathrm{min}$ até $400^{\circ} \mathrm{C}$, por 1 hora, seguida de um aquecimento de $8^{\circ} \mathrm{C} / \mathrm{min}$ até 1200 e 1250 ${ }^{\circ} \mathrm{C}$, com atmosfera controlada de chumbo, em forno Thermolyne 46200, do IPqM. As amostras foram sinterizadas em cadinhos herméticos de alumina para manter uma atmosfera controlada de chumbo.

As cerâmicas PZT I, em ambas as temperaturas de sinterização, de $1200^{\circ} \mathrm{C}$ e de $1250^{\circ} \mathrm{C}$, apresentaram o valor de densidade de cerca de $96 \%$ da densidade teórica. Já as de PZT III, apresentaram valores de densidade de $75 \%$ e de $85 \%$ da densidade teórica nas sinterizações a $1200^{\circ} \mathrm{C}$ e $1250^{\circ} \mathrm{C}$, respectivamente. Cabe lembrar, que, para ambos os pós, em todas as temperaturas, o tempo de sinterização foi de 3 horas. 
$O$ ensaio de dureza foi realizado em um aparelho medidor de micro dureza vickers. 0 equipamento utilizado foi um micro durômetro modelo DHV 1000 da marca Time, do UniFOA. 0 tempo de carregamento foi de 15s com as cargas de 500 e $1000 \mathrm{gf}$, com o uso de um indentador diamantado com formato de uma pirâmide de base quadrada, com $136^{\circ}$ entre as faces.

Os corpos de prova resultantes das sinterizações foram caracterizados por DRX e por MEV. Nas análises de DRX, o tempo de coleta das amostras sinterizadas foi de $5 \mathrm{~s}$ por ponto de contagem, com passo angular de $0,05^{\circ}$. Foi utilizado um difratômetro Shimadzu, modelo XRX 6100, do UniFOA, com radiação CuKa de comprimento de onda $1,5453 \AA$, com uma tensão de tubo de $60 \mathrm{kV}$, corrente de 1,5 A e varredura com $2 \theta$ entre 10 e $80^{\circ}$.

Nas análises de MEV o corpo de prova foi fraturado e uma parte fixada com uma fita de carbono em um suporte metálico. Foi utilizada uma fita de cobre que conectou a amostra com o suporte metálico, a fim de promover a condutividade elétrica. A análise foi realizada com o uso de elétrons secundários em um MEV da marca Hitachi e modelo TM 3000, do UniFOA. As análises por DRX das amostras sinterizadas foram realizadas nas mesmas condições das dos corpos verdes.

\section{$3 \quad$ RESULTADOS E DISCUSSÕES}

A FIG. 1 apresenta os resultados de dureza por micro indentação Vickers do PZT I e III. Os resultados de dureza do PZT I foram maiores em relação aos do PZTIII, nas duas condições de sinterização, 125 e $393 \mathrm{HV}$ e 107 e $126 \mathrm{HV}$, respectivamente. Esses percentuais demonstram que a densificação (GERMAN, 1996; REED, 1995) ocorrida nas amostras de PZT I foi mais eficaz, quando comparada aos do PZT III. Por meio das análises das FIG. 2 a 5 (a) e (b), essa afırmativa se comprova, ou seja, as composições do PZT I apresentaram microestruturas mais densas do que as do PZT III.

Figura 1 - Valores de dureza por micro indentação Vickers do PZT I e III
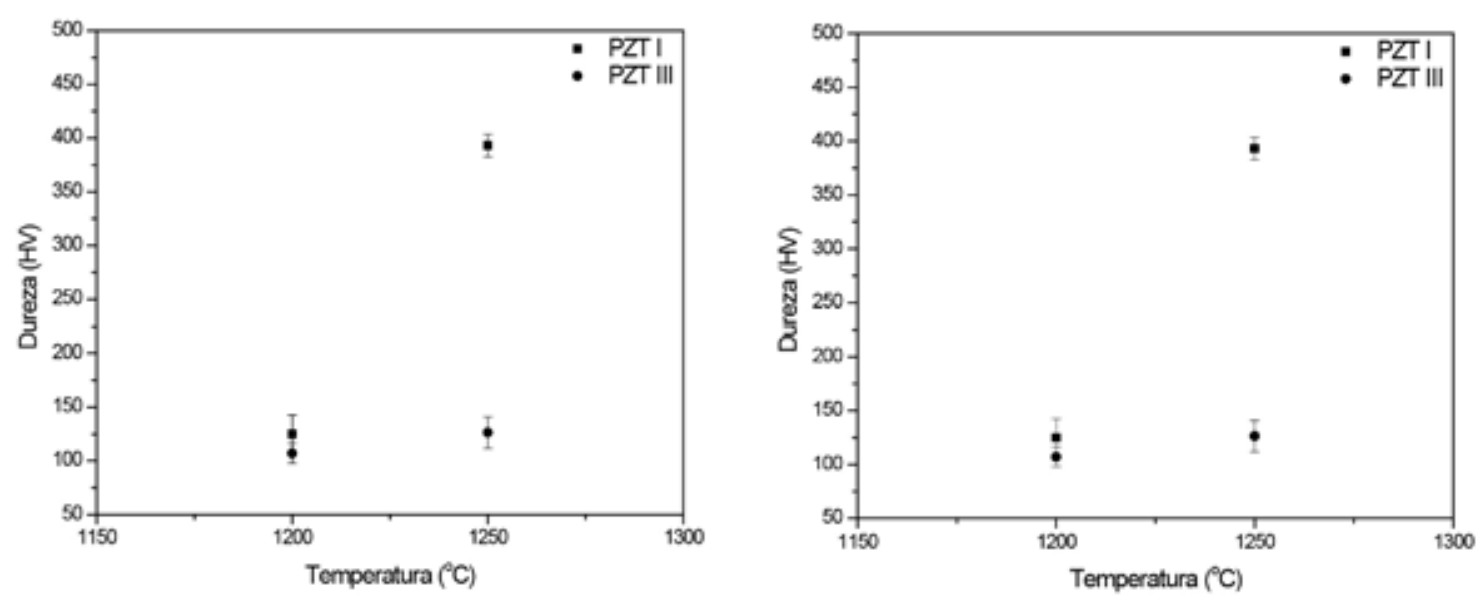

Fonte: dos autores, 2015

Nas Figuras 2 (a) e (b) são mostrados os perfis de difração de raios $X$ dos pós da fase ferroelétrica de PZT.

O pico principal característico do pó de PZT I e III, de maior intensidade, como esperado, foram observados em 2 teta de, aproximadamente, $30^{\circ}$ (Ficha PZT JCPDS\#33-0784). 
Figura 2 - Difratogramas de DRX dos pós de (a) PZT I e (b) PZT III mostram o pico principal característico de PZT em 2 teta igual a $30^{\circ}$.
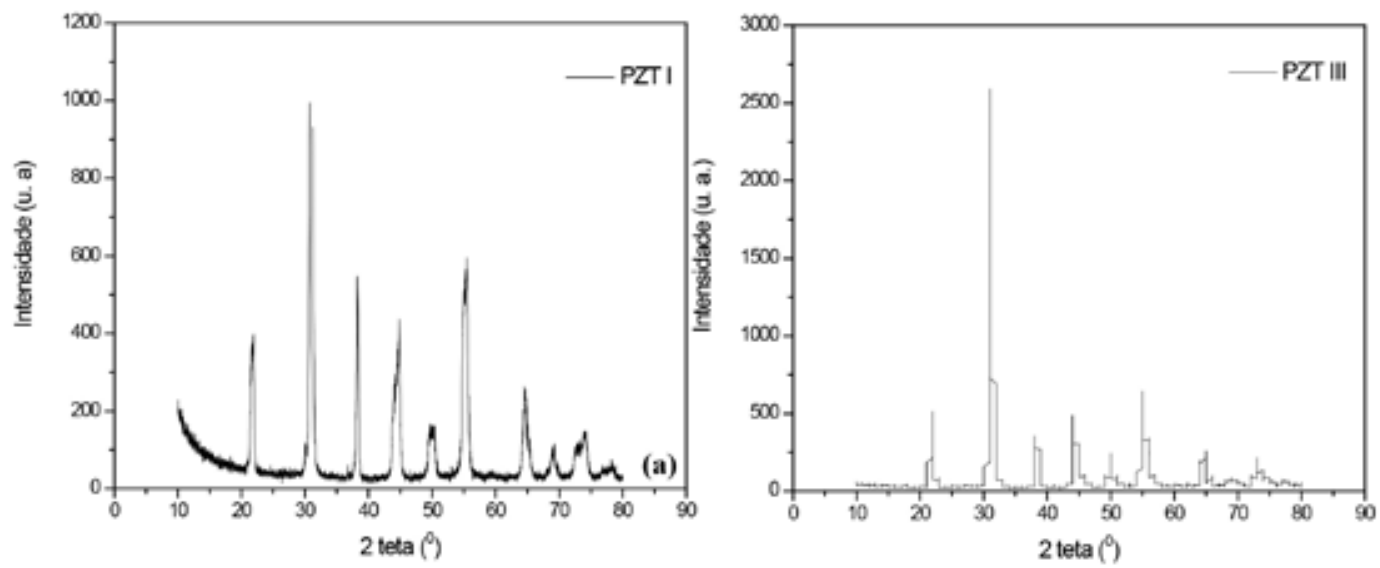

Fonte: dos autores, 2015.

Os perfis de difração de raios $X$ das amostras sinterizadas estão apresentados nas Figuras 3 (a) e (b) e Figuras 4 (a) e (b). As fases esperadas foram observadas como majoritárias em todos os casos. As cerâmicas de PZT I e III apresentaram a coexistência das fases Perovskita com simetria tetragonal e romboédrica, todavia, com traços da fase zircônia.

Observou-se que, nas amostras sinterizadas, o pico principal característico do PZT aparece deslocado para a direita, próximo a 2 teta de $40^{\circ}$ e nota-se também a presença de traços de zircônia próximo a 2 teta de $30^{\circ}$.

Não foram observados traços dessa fase no perfil de DRX dos pós de PZT I e PZT III, o que pode ser, provavelmente, atribuída à perda de $\mathrm{PbO}$ na sinterização, mesmo com o controle de atmosfera rica em óxido de chumbo. A fase indicada com a seta foi indexada por $\mathrm{ZrO}_{2}$ ( ficha JCPDS \#01-0750).

Na figura 3, observam-se picos de mais baixas intensidades com o aumento da temperatura de sinterização: (a) a $1200^{\circ} \mathrm{C} \mathrm{e} \mathrm{(b)} \mathrm{a} 1250^{\circ} \mathrm{C}$

Figura 3 - Difratogramas de DRX do PZT I após sinterização mostram, para ambas as temperaturas, o pico característico de PZT um pouco deslocado para 2 teta próximo a $40^{\circ}$ e traços da fase $\mathrm{ZrO} 2$.
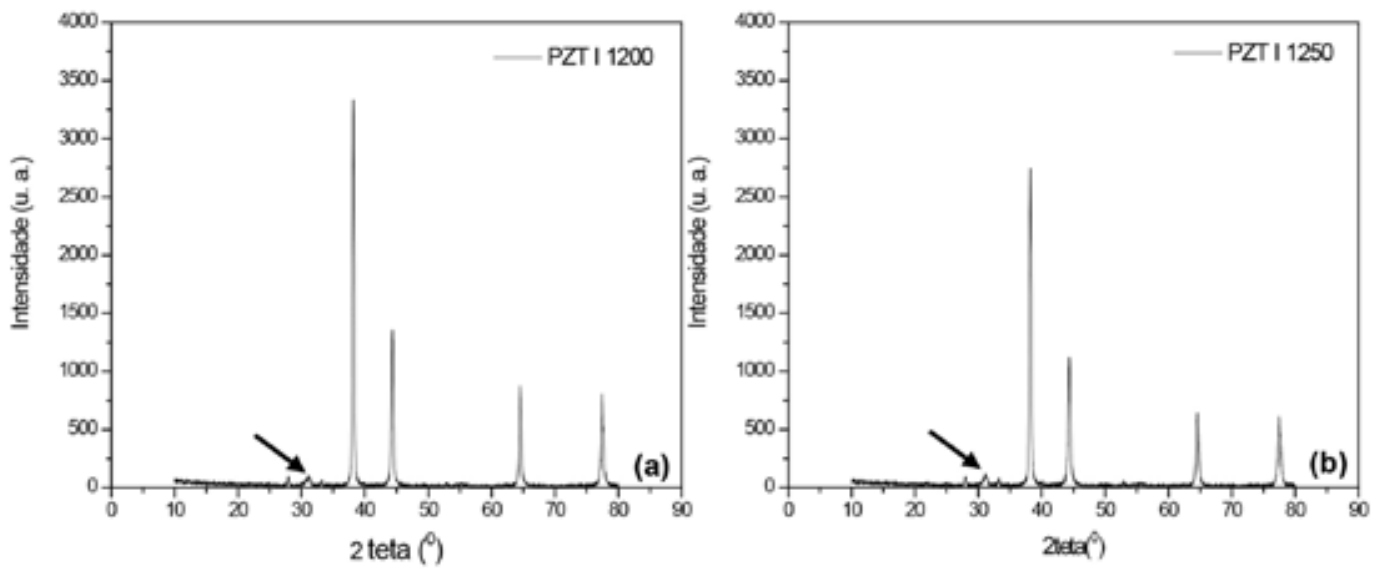

Fonte: dos autores, 2015. 
$\mathrm{Na}$ figura 4, ao contrário do PZT I, observam-se picos de mais altas intensidades com o aumento da temperatura de sinterização: (a) a $1200^{\circ} \mathrm{C} \mathrm{e} \mathrm{(b)} \mathrm{a} 1250^{\circ} \mathrm{C}$.

Figura 4 - Difratogramas de DRX do PZT III após sinterização mostram para ambas as temperaturas, o pico característico de PZT um pouco deslocado para 2 teta próximo a $40^{\circ}$ e traços da fase ZrO2.
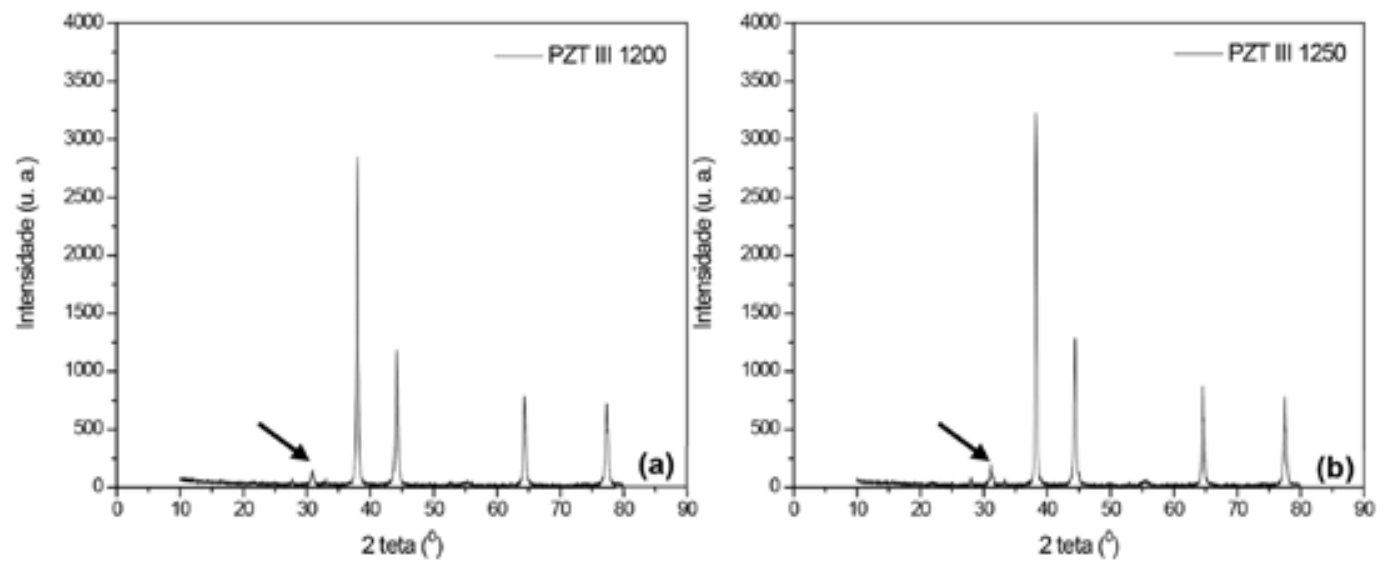

Fonte: dos autores, 2015

Nas Figuras 5 (a) e (b), observam-se as micrografias da amostra de PZT I sinterizada a $1200^{\circ} \mathrm{C}$. Nota-se formação de grãos com formatos equiaxiais, com uma microestrutura densa. Nota-se também, a existência de regiões de aglomerados.

Figura 5 - Amostras sinterizadas a $1200^{\circ} \mathrm{C}$ de PZT I ( (a) $5000 \mathrm{X} \mathrm{e} \mathrm{(b)} 15000 \mathrm{X}$ )
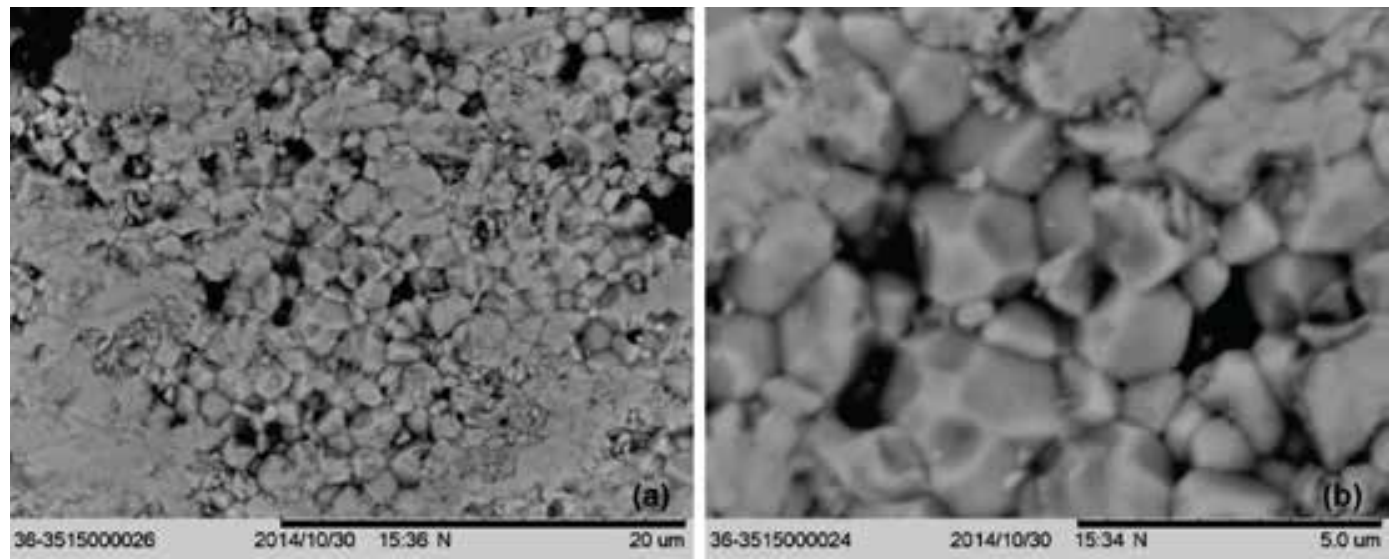

Fonte: dos autores, 2015.

Nas Figuras 6 (a) e (b), foi verificada na microestrutura do PZT I a $1250^{\circ} \mathrm{C}$, maior formação de grãos em tamanhos homogêneos e, ainda, observa-se a presença de alguns aglomerados. 
Figura 6 - Amostras sinterizadas a $1250^{\circ} \mathrm{C}$ de PZT I, sob aumentos de (a) $5000 \mathrm{X}$ e (b) $10000 \mathrm{X}$.
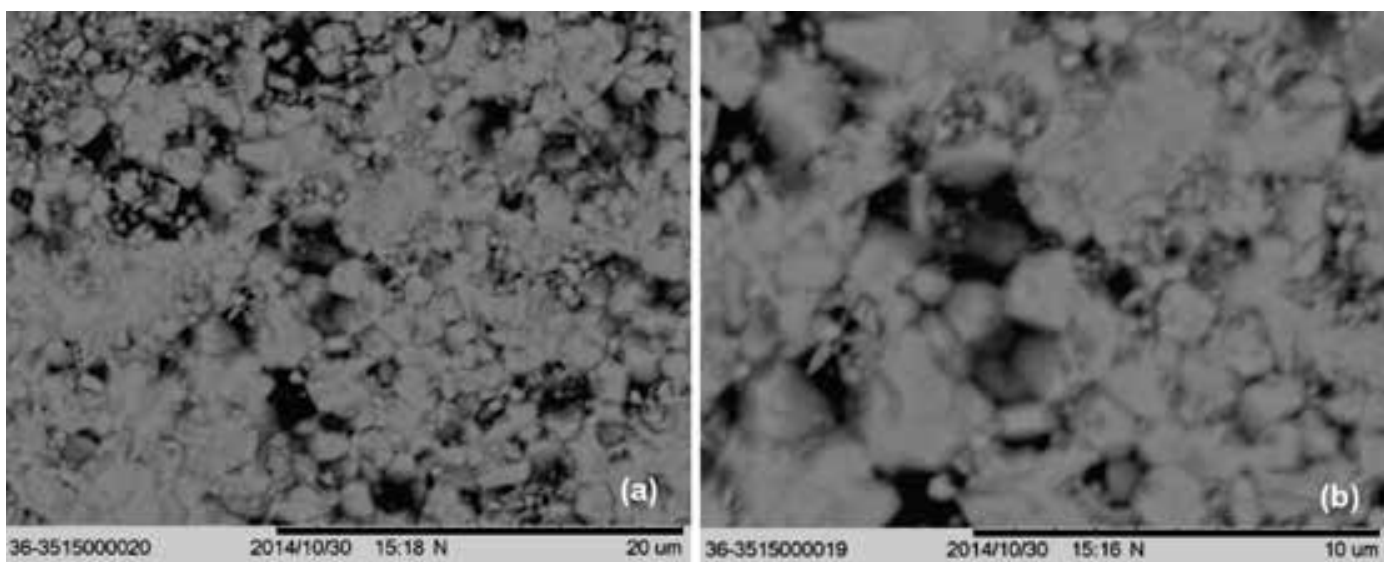

Fonte: dos autores, 2015.

Nas Figuras 7 (a) e (b), observam-se microestruturas de PZT III, onde verifica-se que houve crescimento de grão e muitos poros, o que corrobora com os resultados inferiores de densidade e de dureza em relação aos do PZT I.

Já nas Figuras 8 (a) e (b) na microestrutura do PZT III a $1250^{\circ} \mathrm{C}$, observa-se, também, crescimento de grãos, porém com uma microestrutura mais densa, possivelmente, devido à maior temperatura, 0 que também está de acordo com os resultados de densidade e de dureza.

Figura 7 - Amostras sinterizadas a $1200^{\circ} \mathrm{C}$ de PZT III, sob aumentos de: (a) $5000 \mathrm{X} \mathrm{e}$ (b) $10000 \mathrm{X}$.
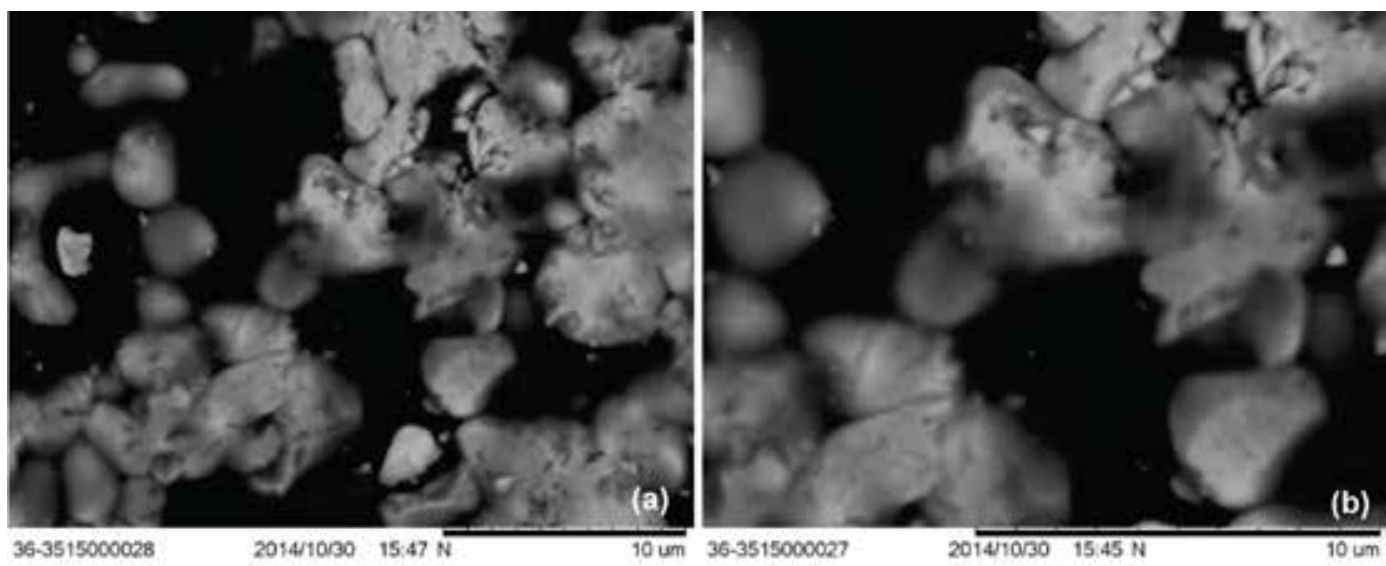

Fonte: dos autores, 2015. 
Figura 8 - Amostras sinterizadas a $1250^{\circ} \mathrm{C}$ de PZT III, sob aumentos de: (a) $10000 \mathrm{X}$ e (b) $15000 \mathrm{X}$.
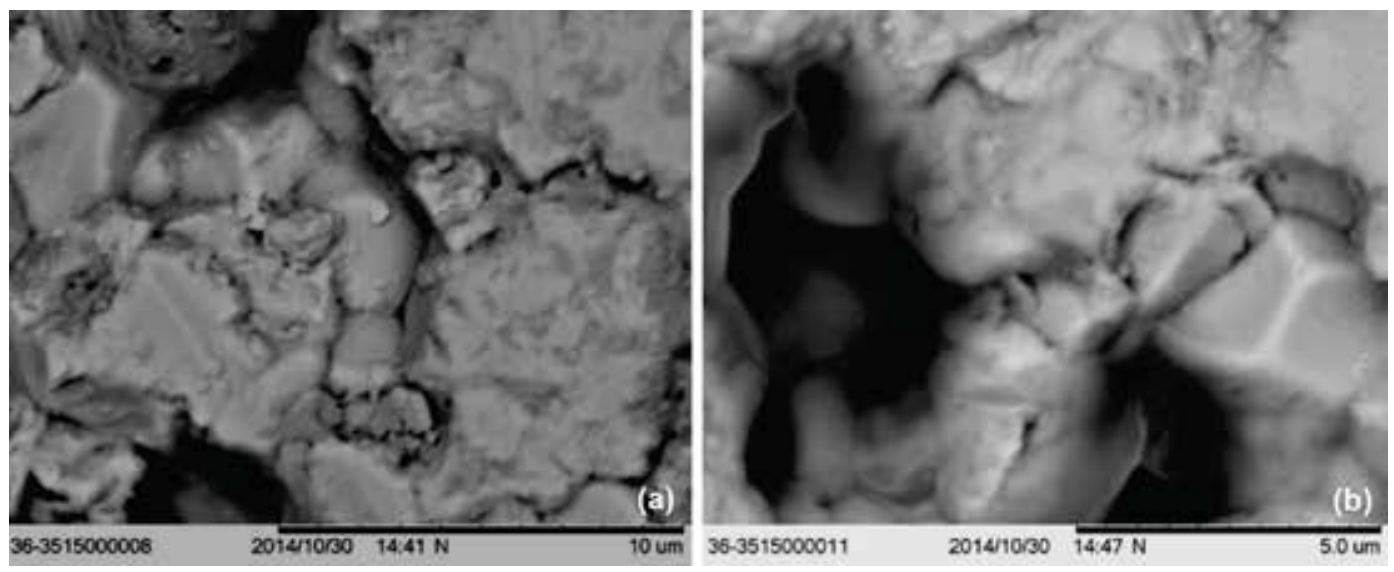

Fonte: dos autores, 2015

\section{CONCLUSÕES}

A cerâmica de PZT I, com densidade de $96 \%$ da densidade teórica, sinterizada a $1250^{\circ}$, apresentou o mais elevado valor de dureza, de 393HV.

O PZT III sinterizado a $1200^{\circ} \mathrm{C}$ apresentou baixo valor de densidade, cerca de $75 \%$ da densidade teórica. Esse valor pode ser corroborado pela microestrutura, onde pode observar-se grandes vazios (porosidades).

A microestrutura do PZT III sinterizado a $1250^{\circ} \mathrm{C}$, embora apresente maior densificação, em relação ao PZT III sinterizado a $1200^{\circ} \mathrm{C}, 84 \%$ da densidade teórica, apresenta regiões de grande aglomerados e observa-se crescimento de grãos facetados.

Em todas as cerâmicas PZT I e III sinterizadas, os difratogramas mostram para ambas as temperaturas, o pico característico de PZT um pouco deslocado para 2 teta próximo a $40^{\circ}$.

Não foi observado traços da fase ZrO2 no perfil de DRX dos pós de PZT I e PZT III. A presença dela nas cerâmicas pode ser, provavelmente, atribuída à perda de $\mathrm{PbO}$ na sinterização, mesmo com o controle de atmosfera rica em óxido de chumbo.

\section{AGRADECIMENTOS}

Os autores agradecem ao Centro Universitário de Volta Redonda (UniFOA) e ao Instituto de Pesquisa da Marinha (IPqM), pelo apoio dado ao desenvolvimento deste projeto. 


\section{REFERÊNCIAS}

FESENKO, E. G.; OLGA, RAZUMOVSKAYA, N.. Influence of The Chemical Composition on the Physical Properties of PZT -TYPE Piezoceramic Transducers. Molecular and Quantum Acoustics, v. 24, 2003.

GERMAN, R. M. Sintering Theory and Pratices, John Wiley \& Sons, 1996.

GOLJAHI S.; LYNCH, C. S. Effects of electric field on the fracture toughness $\left(K_{\mathrm{IC}}\right)$ of ceramic PZT. Smart Materials and Structures, v. 22, n. 9, 2013.

KHALID, M.; SHOAIB, M.; KHAN, A.. Strontium doped lead zirconate titanate ceramics: study of calcination and sintering process to improve piezo effect. Journal Nanoscience Nanotechnol, v. 11, n. 6, p. 5440$5445,2011$.

KOUR, P.; SINHA, S. K.. Studies of $\mathrm{Sr}^{2+}$ ion substitution on ferroelectric and piezoelectric properties of PZT nanocrystalline. Cerâmica, v. 59, p. 34-38, 2013.

NBR 6220, Material refratário denso - Determinação da massa específica aparente, porosidade aparente, absorção e massa específica aparente da parte sólida, ABNT, 1990.

REED, J. S., Principles of Ceramics Processing, John Wiley \& Sons, 1995.

YANG, J. S.; CHEN, X. M., AIZAWA, T., KUWABARA, M.. PZT based piezoelectric ceramics with enhanced fracture toughness. Solid State lonics, v. 108, p. 117-121, 1998.

ZHANG, T. Y.; GAO, C. F. Fracture behaviors of piezoelectric materials. Theoretical and Apllied Fracture Mechanics, v. 41, p. 339-379, 2004. 